\title{
Assessment of interatomic potentials for atomistic analysis of static and dynamic properties of screw dislocations in $\mathrm{W}$
}

\author{
D Cereceda , A Stukowski , M R Gilbert , S Queyreau , Lisa Ventelon , \\ M-C Marinica , J M Perlado' and J Marian
}

\begin{abstract}
Screw dislocations in bcc metals display non-planar cores at zero temperature which result in high lattice friction and thermally-activated strain rate behavior. In bec W, electronic structure molecular statics calculations reveal a compact, non-degenerate core with an associated Peierls stress between 1.7 and $2.8 \mathrm{GPa}$. However, a full picture of the dynamic behavior of dislocations can only be gained by using more efficient atomistic simulations based on semiempirical interatomic potentials. In this paper we assess the suitability of five different potentials in terms of static properties relevant to screw dislocations in pure W. Moreover, we perform molecular dynamics simulations of stress-assisted glide using all five potentials to study the dynamic behavior of screw dislocations under shear stress. Dislocations are seen to display thermally-activated motion in most of the applied stress range, with a gradual transition to a viscous damping regime at high stresses. We find that one potential predicts a core transformation from compact to dissociated at finite temperature that affects the energetics of kink-pair production and impacts the mechanism of motion. We conclude that a modified embedded-atom potential achieves the best compromise in terms of static and dynamic screw dislocation properties, although at an expense of about ten-fold compared to central potentials.
\end{abstract}

(Some figures may appear in colour only in the online journal)

\section{Introduction}

Tungsten (W) is considered as a leading candidate for plasma-facing applications in magnetic fusion energy (MFE) devices. The most attractive properties of $W$ for MFE are its high melting point and thermal conductivity, low sputtering yield and low long-term disposal radioactive footprint. These advantages are accompanied unfortunately by very low fracture toughness characterized by brittle transand inter-granular failure, which severely restricts the useful operating temperature window [1].
Transgranular plasticity in refractory metals, including $\mathrm{W}$, is governed by the temperature dependence of screw dislocation motion. $\mathrm{W}$ is typically alloyed with 5-26 at.\% Re to increase low temperature ductility and improve high temperature strength and plasticity [2]. The physical origins behind the Re-induced ductilization have been discussed in the literature [3-5] and point in some way or another to alterations in the core structure of $\frac{1}{2}\langle 111\rangle$ screw dislocations, which both reduce the effective Peierls stress op and extend the number of possible slip pathways. A direct consequence of a reduced Peierls stress, e.g. as via Re alloying, is an enhanced dislocation mobility at low temperatures. Recent 
electronic structure calculations of $\sigma_{\mathrm{P}}$ in pure $\mathrm{W}$ gave values between 1.7 and $2.8 \mathrm{GPa}[3,6]$. This means that, under most conditions relevant to technological applications, where stresses are of the order of only a few hundred MPa, a reduction in $\sigma_{\mathrm{P}}$ of a few hundred $\mathrm{MPa}$ may not be significant to the plastic behavior of $\mathrm{W}$ and $\mathrm{W}$ alloys. Instead, it is the thermally-activated and three-dimensional character of screw dislocation motion, the associated solution softening behavior, as well as the temperature dependence of the core structure, that control bulk ductility.

None of these aspects can be studied in atomistic detail using current experimental capabilities. By contrast, atomistic methods based on semiempirical potentials have enabled large-scale molecular dynamics (MD) simulations, so that, at present, calculations of single dislocation mobility, core structure and transformations, etc, can be obtained with reasonable accuracy. However, care must be exercised when choosing from the dozen or so $\mathrm{W}$ potentials available in the literature. Semiempirical force fields with both pair and cohesive contributions (e.g. following the embedded-atom method formalism) are typically considered to achieve an optimum balance between efficiency and accuracy. These are typically fitted to reproduce some basic bulk and defect properties such as lattice parameter, elastic constants, vacancy formation energy, surface energies, etc, but generally not dislocation properties. Of these, it is known that the screw dislocation core structure at $0 \mathrm{~K}$ should be non-degenerate (also known as compact), as revealed by density functional theory (DFT) calculations $[3,5,6]$.

Previous atomistic calculations of screw dislocations in W have been performed by Mrovec et al [7], Fikar et al [8] and Tian and Woo [9]. Mrovec et al studied the dislocation core structure and calculated the Peierls stress at $0 \mathrm{~K}$ using a tight-binding-based bond-order potential (TB-BOP) ${ }^{5}$. They predicted a non-degenerate core structure and a Peierls stress of $4.3 \mathrm{GPa}$. For their part, Fikar et al studied core structures and energies of screw dislocations using three different interatomic potentials, all of which displayed dissociated cores. Lastly, Tian and Woo examined the mobility of screw dislocations also with an embedded-atom potential that predicted a dissociated core structure. They were able to obtain dislocation velocities at stresses above the Peierls stress at $0 \mathrm{~K}$. However, no systematic study of dislocation motion in $\mathrm{W}$ at finite temperature has been conducted. Characterization of dislocation motion in the stress-temperature space is important to parameterize the so-called mobility functions used in higher-level methods such as dislocation dynamics. The purpose of the mobility functions is to provide a quantitative measure of the response of dislocations to applied and internal stresses.

Unfortunately, one of the most important difficulties associated with such studies is the scale-dependent nature of MD simulations, which require exceedingly high strain rates to drive the system over time scales accessible computationally, of the order of a few tens of nanoseconds.

\footnotetext{
5 Bond-order potentials include non-central atomic interactions to represent the effect of d-electrons in transition metals.
}

Because of these limitations, MD is incapable at present of properly capturing the thermally-activated motion of screw dislocations at low stresses. However, MD simulations can still provide valuable input in intermediate-to-high stress conditions and in situations where the deformation rates are high. The objective of this paper is to compare five different interatomic potentials - that have not been fitted against screw dislocation data - and assess their performance in terms of static and dynamic screw dislocation properties. By static properties we mean several reference parameters at $0 \mathrm{~K}$ as obtained with DFT calculations. The dynamic behavior is evaluated in terms of screw dislocation mobility as a function of stress and temperature. Due to the absence of 'reference' mobility data against which to compare the potentials, we will simply draw several general conclusions based on the inter-comparison among potentials.

The paper is organized as follows. First, we discuss the distinctive features of each potential and calculate the structure of a screw dislocation core. The Peierls potential and the $\gamma$ surface are then calculated and verified against existing DFT and TB-BOP calculations. Following this, we introduce the computational setup for the dynamic mobility simulations and calculate dislocation velocities as a function of temperature and stress. Subsequently, a study of the core trajectories in the plane defined by the glide and normal directions is carried out. We finish by analyzing the causes of the temperature-dependent behavior of each potential and emphasizing the insufficiency of static calculations to fully characterize dislocation motion at finite temperatures.

\section{Computational details}

\subsection{Interatomic potentials}

Our calculations were performed with the parallel MD code LAMMPS [15]. Table 1 gives basic information about the five different potentials considered here, among which there are three embedded-atom method (EAM) potentials, one Tersoff-Brenner-type bond-order potential (TF-BOP) and one modified EAM (MEAM). Note that the TB-BOP used by Mrovec et al [7] was deemed not suitable for dynamics simulations by its authors [10] and has thus not been considered here. Hereafter, these potentials are referred to in the text by the identifiers given in the table header. This selection of $\mathrm{W}$ potentials, from the dozen or so available in the literature, is not meant to be an implicit assessment of the quality of those not employed here.

Two important quantities for the characterization of screw dislocation cores at $0 \mathrm{~K}$ are the Peierls potential, defined as the energy path from one equilibrium position to another on a $\{110\}$ plane, and the $\gamma$ surface along the [111] direction also on $\{110\}$ planes. The Peierls potential governs the morphology of kinks (e.g. [21]), while workers such as Duesbery and Vitek [22] have provided evidence for a direct correspondence between the shape of the $\frac{1}{2}\langle\mathbf{1 1 1}\rangle\{110\}$ gamma surface and the screw dislocation core structure. These are plotted, respectively, for all potentials in figures 1 and 2 on the (110) plane. DFT data for both calculations are also shown for comparison. 
Table 1. The properties of the potentials used: lattice parameter $a_{0}$, shear modulus $\mu$, Peierls stress $\sigma_{\mathrm{P}}$, computational cost, core structure at $0 \mathrm{~K}$ and thermal expansion coefficient $\alpha$. Potentials EAM1, EAM2 and TF-BOP display a three-fold symmetric (degenerate) core, while EAM3 and MEAM predict compact (non-degenerate) cores. The values of the volumetric thermal expansion coefficients, $\alpha$, are used in section 3.3

\begin{tabular}{llllll}
\hline Potential & EAM1 & EAM2 & EAM3 & TF-BOP & MEAM \\
\hline Reference & {$[17]$} & {$[18]$} & a & {$[19]$} & {$[20]$} \\
$a_{0}(\AA)$ & 3.165 & 3.165 & 3.143 & 3.165 & 3.188 \\
$\mu(\mathrm{GPa})^{\mathrm{b}}$ & 160 & 163 & 161 & 170 & 161 \\
$\sigma_{\mathrm{P}}(\mathrm{GPa})^{\mathrm{c}}$ & 4.0 & 3.9 & 1.8 & 1.1 & 3.2 \\
Computational $^{\mathrm{a}}$ & 1.0 & 0.4 & 0.9 & 5.4 & 9.1
\end{tabular}

cost relative to

EAM1 ${ }^{\mathrm{d}}$

Core structure at $0 \mathrm{~K}^{\mathrm{e}}$
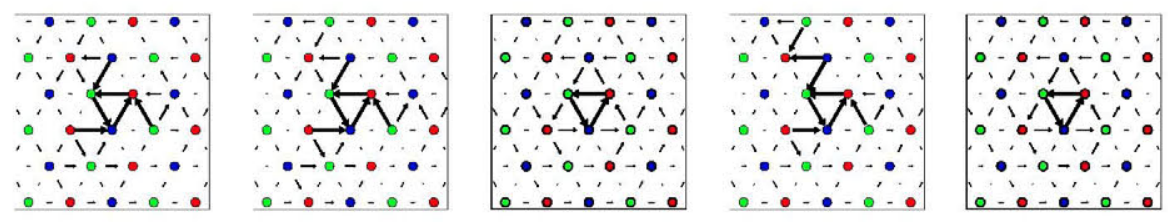

$\alpha\left(\times 10^{-5} \mathrm{~K}^{-1}\right)^{\mathrm{f}} \quad 1.40$

2.42

1.76

2.38

1.64

a This potential was fitted in the same fashion as the M07 potential for $\mathrm{Fe}$ given in [35]. The potential is based on the force matching method of Ercolessi-Adams [36] and includes fitting data from two main sources. The first includes perfect crystal experimental properties, such as the lattice constant and the elastic constants. The second source includes DFT-calculated defect properties as well as force fields associated with several liquid W configurations. Using this procedure, several converged sets of parameters are produced, but only those parameterizations yielding the desired dislocation properties are retained.

${ }^{\mathrm{b}} \mathrm{W}$ is isotropic elastic and, thus, the value of $\mu$ given is equally valid for $\{110\}$ and/or $\{112\}$ slip.

${ }^{c}$ For consistency, our Peierls stress calculations use the same geometry as the DFT calculations by Romaner et al [3] and Samolyuk et al [6], which reveal a value of $\sigma_{\mathrm{P}}$ between 1.7 and $2.8 \mathrm{GPa}$.

${ }^{d}$ The computational cost was evaluated using the cutoff radii specified in the original reference for each potential.

${ }^{\mathrm{e}}$ DFT calculations predict a compact, non-degenerate core.

${ }^{\mathrm{f}}$ The experimental value for $\alpha$ is $1.45-1.91 \times 10^{-5} \mathrm{~K}^{-1}$ in the $1000-2000 \mathrm{~K}$ temperature interval [16].

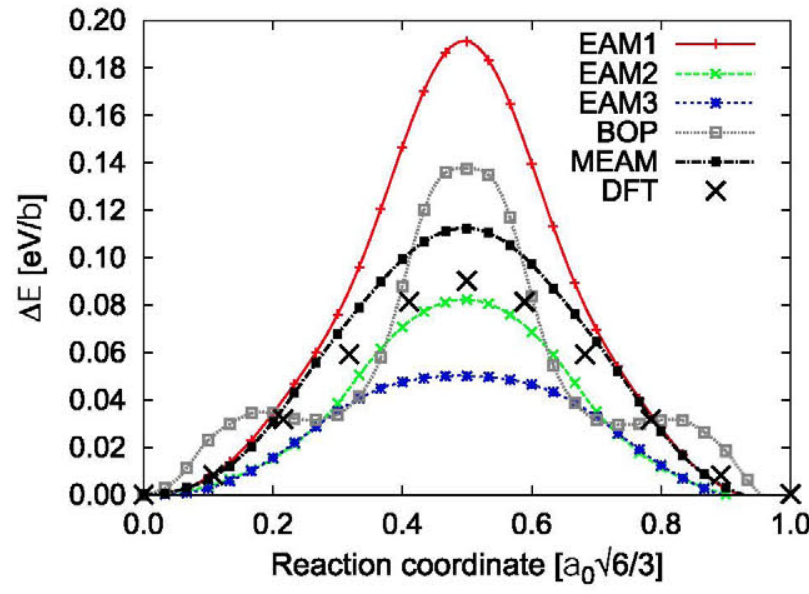

Figure 1. The Peierls potential for all potentials tested here. DFT calculations from Ventelon et al [26] are shown for comparison.

The Peierls potential was obtained using the nudged elastic band method [23] in the manner proposed by Gröger and Vitek [24], whereas the DFT calculations in both cases were obtained using a plane wave self-consistent field code as described in $[25,26]$.

\subsection{Simulation setup}

To measure dislocation velocities, we performed stresscontrolled simulations of $\frac{1}{2}\langle 111\rangle$ dislocations with the

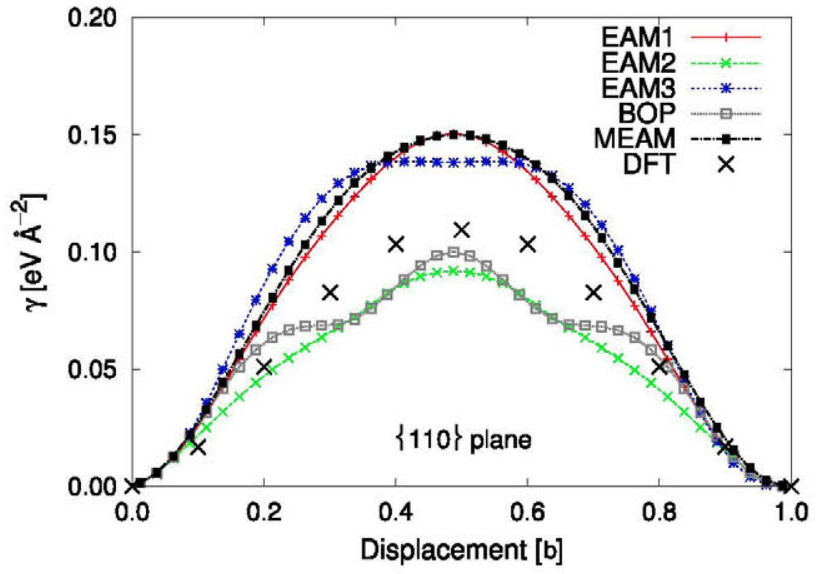

Figure 2. The $(1 \overline{1} 0) \gamma$ surface along the [111] direction for all the potentials considered in this work. DFT calculations from Ventelon et al [26] are shown for comparison.

maximum resolved shear stress (MRSS) on a $\{112\}$ plane. The justification for focusing on $\{112\}$-type planes is twofold. First, as Argon and Maloof [11] have shown, under tensile loading most loading orientations and temperatures result in some degree of $\frac{1}{2}\langle 111\rangle\{112\}$ slip. Also, Li et al [5] have shown that $\{112\}$ slip is important in W alloys with high Re concentrations. Second, certain EAM potentials intrinsically deviate from MRSS behavior when the MRSS plane is of the 

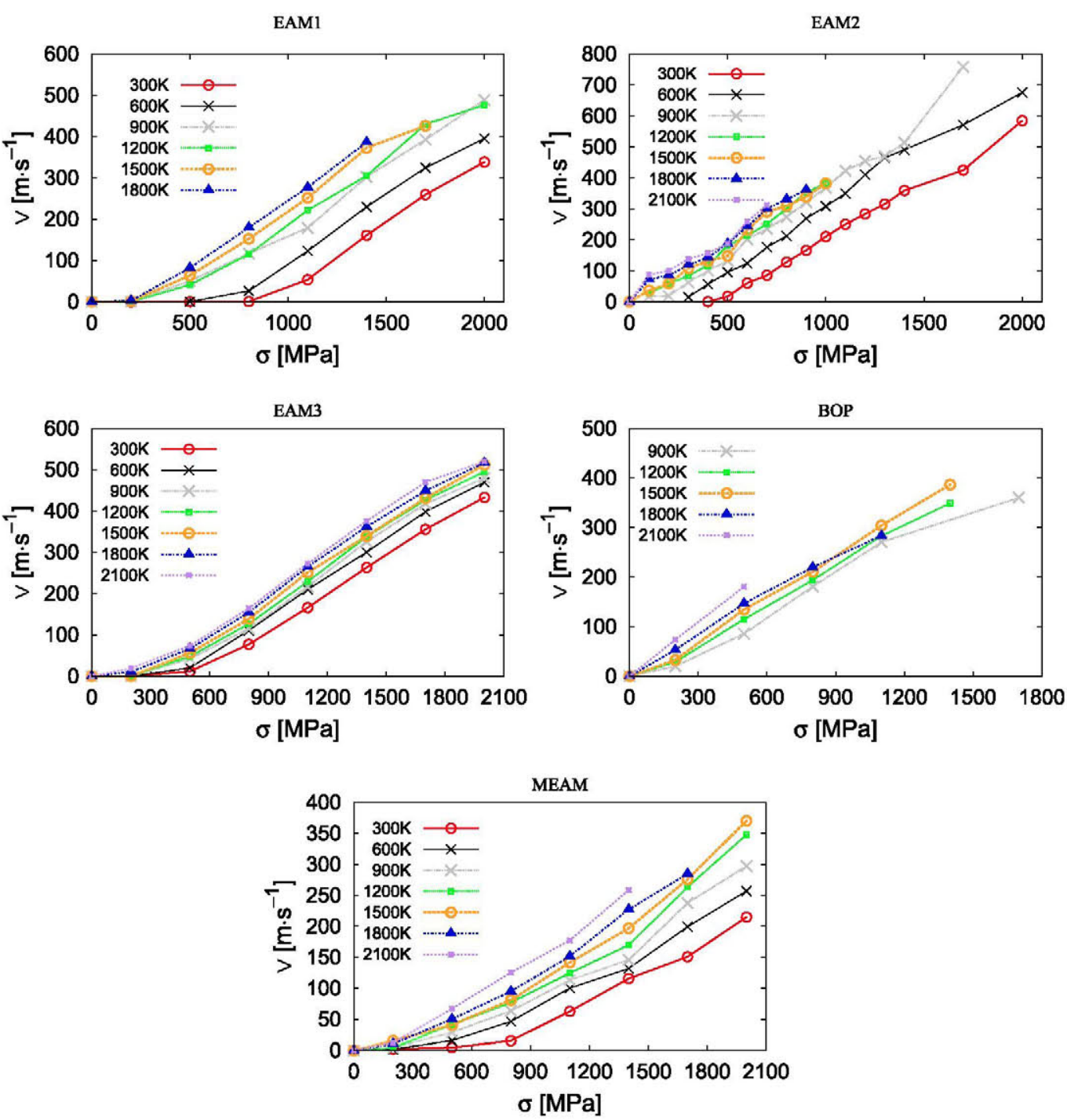

Figure 3. The screw dislocation velocity as a function of the applied shear stress and temperature for the five interatomic potentials considered here. Note that the velocity and stress axes are not on the same scale for each case.

$\{110\}$ type for reasons that have been discussed at length in the literature $[12,13,29]$.

We have provided the details regarding the computational setup for this type of simulation in prior publications [13, 14]. Suffice it to say that orthogonal boxes of sufficient size with axes $x \equiv \frac{1}{2}[111], y \equiv[1 \overline{1} 0]$ and $z \equiv[11 \overline{2}]$, corresponding to the line, glide and plane normal directions, respectively, were used to mitigate finite size effects (cf [13]). $\sigma_{x z}$ was then applied on the computational cell boundaries and simulations were conducted in the NPT ensemble. Periodic boundary conditions were used in the line and glide directions. The reference cell dimensions were $L_{x}=$ $25\left[\frac{\sqrt{3}}{2} a_{0}\right], L_{y}=100\left[\sqrt{2} a_{0}\right]$ and $L_{z}=50\left[\sqrt{6} a_{0}\right]$, where the amounts in brackets are the dimensions of the nominal unit cell in the coordinate system employed here. The reference configuration contained $7.5 \times 10^{5}$ atoms, which resulted in strain rates of $1.4 \times 10^{6-7} \mathrm{~s}^{-1}$ for dislocation velocities between 10 and $100 \mathrm{~m} \mathrm{~s}^{-1}$.
All simulations were run on LLNL's ATLAS cluster using 128 and 256 processors at a reference cost of $1.7 \times 10^{-5}$ CPU seconds per atom per time step for potential EAM1.

\section{Results}

The simulation setup, boundary conditions and velocity calculations, as they relate to the present work, are discussed in depth by Cereceda et al [14]. The temperature and stress ranges covered were, respectively, 300-2100 K and $200-2000 \mathrm{MPa}$. All the simulations were run for $100 \mathrm{ps}$ and configuration data were extracted every picosecond. The procedure to extract dislocation velocities from MD simulations is well established in the literature [13, 27, 28]: from the position of the core, velocities are calculated as the derivative of the displacement-time curve for each case.

\subsection{Screw dislocation mobility}

Figure 3 shows all the $(\sigma-v)$ data for the five interatomic potentials tested. The figure also contains the temperature 
dependence for each case. Generally, the velocities increase monotonically with stress and temperature, although at different rates depending on the potential. To first order, the mechanism of motion followed by the dislocations depends on the Peierls stress. This means that, at a maximum applied shear stress of $2000 \mathrm{MPa}$, the EAM1, EAM2 and MEAM potentials operate under $\sigma_{\mathrm{P}}$ (cf table 1), while for the EAM3 and TF-BOP there are several data points above it. In either case, dislocation motion is mostly governed by the thermally-activated kink-pair nucleation mechanism, and thus displays an exponential dependence with $\sigma$ and $T$. This can be qualitatively appreciated in the figure, although in the appendix a more quantitative analysis is carried out.

Another important aspect of dislocation motion is the extent of MRSS motion displayed, i.e. whether there are deviations from glide on the MRSS \{112\}-type plane. In figure 4 we analyze the trajectories on the $y z$ plane for different combinations of $\sigma$ and $T$ over $100 \mathrm{ps}$ of simulation. Perfect MRSS behavior is characterized by trajectories parallel to $0^{\circ}$. As the figure shows, all the EAM potentials display nearly perfect MRSS behavior, while for the MEAM small deviations in the acceleration phase are captured. The TF-BOP potential displays the most erratic motion, with an overall deviation of the order of $5^{\circ}$. At lower temperatures, this effect is enhanced to the point that the dislocation exits the simulation box only a few picoseconds after the shear stress is applied. This is the reason why there are fewer data points - and none below $900 \mathrm{~K}$ - in the $\sigma-v$ curves shown in figure 3 for the TF-BOP potential. In any case, the trajectories shown in figure 4 are effective, i.e. they are not sufficiently time resolved to capture the atomistic details of dislocation motion. Nevertheless, the operating mechanism of motion is by way of nucleation and propagation of kink pairs on $\{110\}$ planes adjacent to the MRSS (112) plane.

\subsection{Dislocation core structure at finite temperature}

As shown in figure 3 , the $\sigma-v$ data are not conducive to comparison among potentials. Instead, in figure 5 they are plotted as a function of interatomic potential for a number of selected temperatures. The figure reveals an interesting trend: the relative behavior of all the potentials remains unchanged for all temperatures with the exception of EAM3. At low temperatures, this potential exhibits a relatively high dislocation mobility, akin to that displayed by 'fast' potentials such as EAM2. However, above $900 \mathrm{~K}$, the mobility is reduced (relative to the other interatomic potentials) to values more in line with 'slower' potentials such as EAM1. Moreover, if one examines the trajectories followed by the dislocation at $500 \mathrm{MPa},{ }^{6}$ a notable difference in behavior within the EAM3 potential can be observed. At a temperature of $600 \mathrm{~K}$, the dislocation follows a biased path on an effective glide plane forming $\approx 30^{\circ}$ with the MRSS plane. However, at $1800 \mathrm{~K}$, the dislocation follows a path that deviates only

\footnotetext{
6 To be meaningful, this analysis must be performed at relatively low stresses to interfere with the investigated temperature effect by the least amount possible.
}
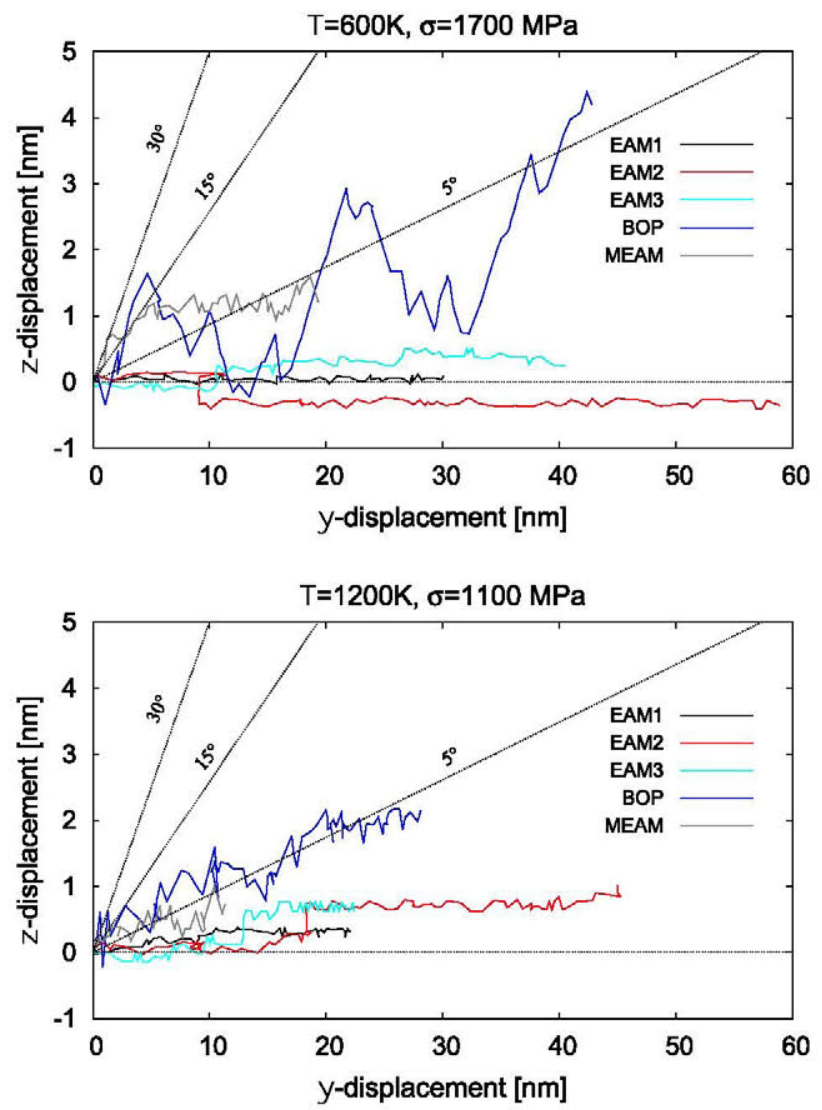

Figure 4. Line-averaged dislocation trajectories on the $y z$ plane for two combinations of $\sigma$ and $T$ and over $100 \mathrm{ps}$. Planes forming $0^{\circ}$, $5^{\circ}, 15^{\circ}$ and $30^{\circ}$ with the (112) MRSS plane are represented with dotted lines (angles not to scale). Except for the TF-BOP potential, all the simulations yield small $<5^{\circ}$ deviations from MRSS motion.

slightly from that dictated by the Peach-Köhler force (i.e. $0^{\circ}$ ). This is quantitatively displayed in figure 6 , where this time the trajectories are resolved with atomistic detail. The figure shows unequivocally that dislocation motion proceeds via the formation of kink pairs on $\{110\}$ planes bordering the MRSS $[11 \overline{2}]$ plane (at $\pm 30^{\circ}$ ). Moreover, the details of the trajectory at $600 \mathrm{~K}$ suggest biased formation on the $(10 \overline{1})$ plane $\left(+30^{\circ}\right)$, whereas at $1800 \mathrm{~K}$ random-walk behavior is displayed, with kink pairs forming equally on both available $\{110\}$ planes.

The behaviors illustrated in figures 5 and 6 for the EAM3 potential suggest a change in core structure with temperature for a given stress state ${ }^{7}$. To examine the physical structure of the dislocation core at different temperatures one can use time-averaged differential displacement (DD) maps (these maps were used in table 1 for each $0 \mathrm{~K}$ configuration). The DD maps are obtained by running MD simulations of crystals containing four screw dislocations arranged in a balanced quadrupole configuration with periodic boundary conditions. The size of the simulation box is $20 \times 15 \times 18$ multiples of the bec lattice vectors $[111] \times[\overline{1} 2 \overline{1}] \times[\overline{101}]$. The dimensions are adjusted to the equilibrium lattice constant at the given temperature. For the finite temperature simulations, the 7 We know that stress also induces its own core transformations, as explained
in [37]. 

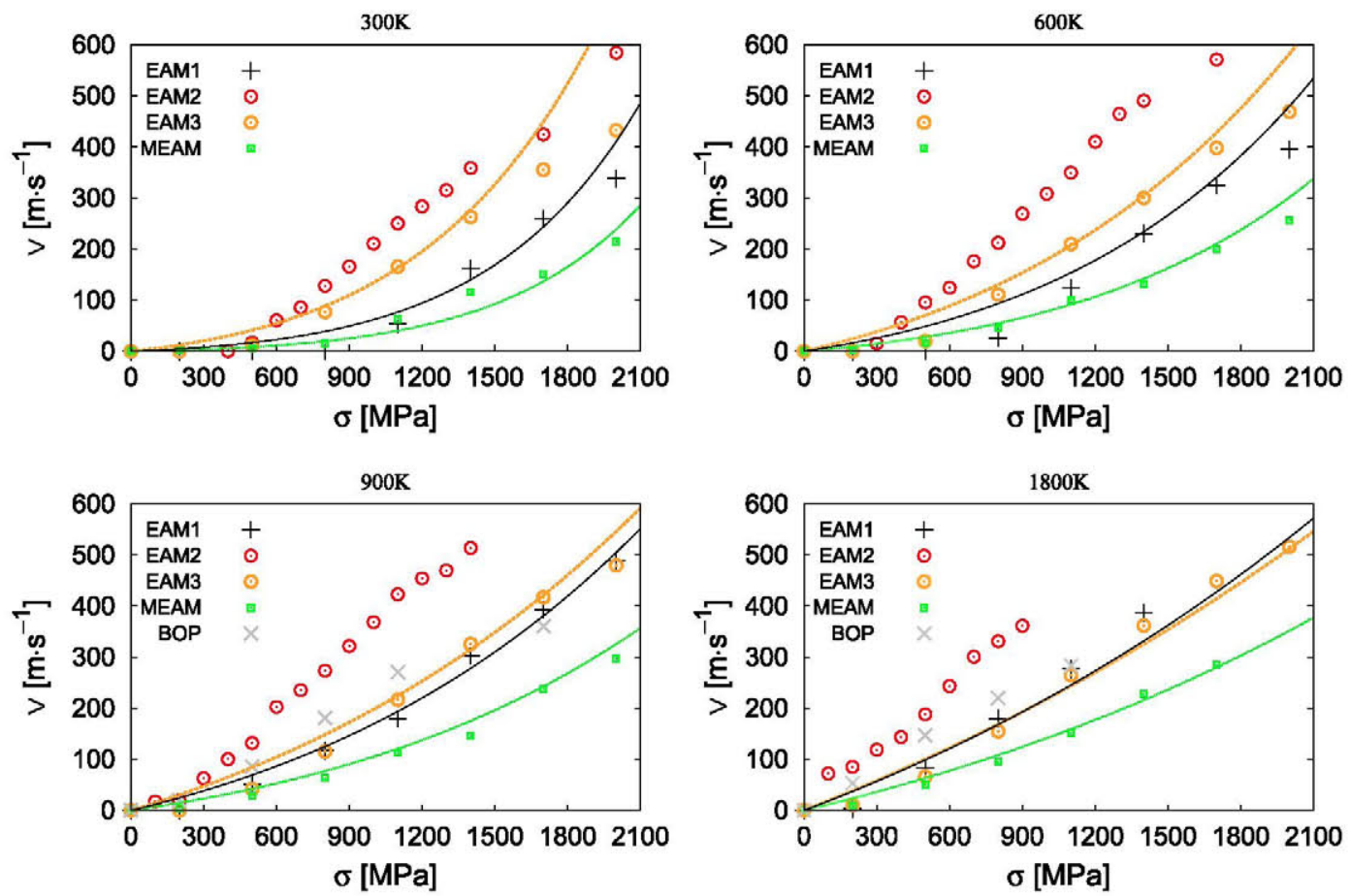

Figure 5. Comparison of interatomic potentials for the data given in figure 3 . The colored lines correspond to the exponential fits obtained in the appendix for the EAM1, EAM3 and MEAM potentials.

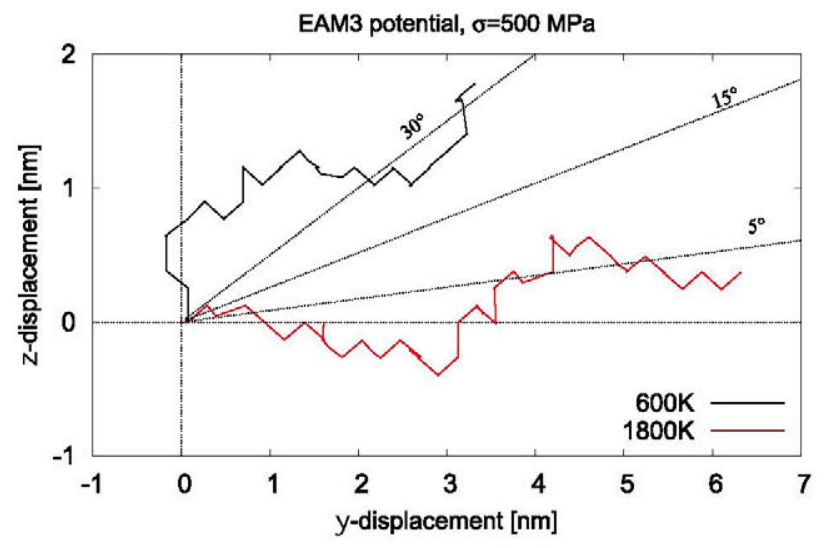

Figure 6. Line-averaged dislocation trajectories on the $y z$ plane for the EAM3 potential at $500 \mathrm{MPa}$. Results for two $200 \mathrm{ps}$ temperatures above and below the presumed core transformation temperature of around $1200 \mathrm{~K}$ are shown. Planes forming $0^{\circ}, 5^{\circ}$, $15^{\circ}$ and $30^{\circ}$ with the $(11 \overline{2})$ MRSS plane are represented with dotted lines (angles not to scale).

displacement of each atomic string is determined by averaging over all 40 atoms in the string and over a time window of $100 \mathrm{fs}$, this being sufficiently long to avoid noise due to thermal vibrations yet short enough to not capture diffusive behavior. The results are shown in figure 7 for configurations in the $0 \mathrm{~K}<T<2100 \mathrm{~K}$ interval. The figure confirms that the EAM3 core is the only one showing an appreciable transformation from non-degenerate to degenerate, clearly seen at and above $1500 \mathrm{~K}$. Although DD maps are a useful tool to quickly analyze core structures, next we complement the results in figure 7 with a more quantitative approach based on fundamental lattice properties.

\subsection{Analysis of screw dislocation core stability}

Duesbery and Vitek [22] have provided a simple rule that relates the shape of the $\frac{1}{2}\langle 111\rangle\{110\} \gamma$-surface to the core structure at $0 \mathrm{~K}$. They used the inequality

$$
\gamma\left(\frac{b}{3}\right)>2 \gamma\left(\frac{b}{6}\right)
$$

to predict whether a screw dislocation will display a compact core. $\gamma\left(\frac{b}{3}\right)$ and $\gamma\left(\frac{b}{6}\right)$ are the energies corresponding to the $\frac{b}{3}$ and $\frac{b}{6}$ magnitudes of the generalized fault vectors, which can be obtained by reference to figure 2 . The idea is that, if the above inequality is satisfied, $\frac{b}{6}$-type faults will be preferred over $\frac{b}{3}$ ones, leading to non-dissociated core structures. However, although Duesbery and Vitek applied this simple rule to six different bec metals ${ }^{8}$ with remarkable success, we find that in our case it does not hold for potentials EAM1 and EAM2. Thus, here we try a different approach based on the analysis carried out by Gilbert and Dudarev [30].

These authors have shown that, alternatively, the $\frac{1}{2}\langle 111\rangle$ screw dislocation core structure in bec systems can be related to the periodic interaction energy between adjacent $\langle 111\rangle$ strings of atoms forming the crystal. Their analysis, which was performed primarily to help guide potential development, provides a framework to predict whether the favored core structure at $0 \mathrm{~K}$ is compact or dissociated. In

$8 \mathrm{~V}, \mathrm{Cr}, \mathrm{Nb}, \mathrm{Mo}$, Ta and W, all described by Finnis-Sinclair potentials [38]. 


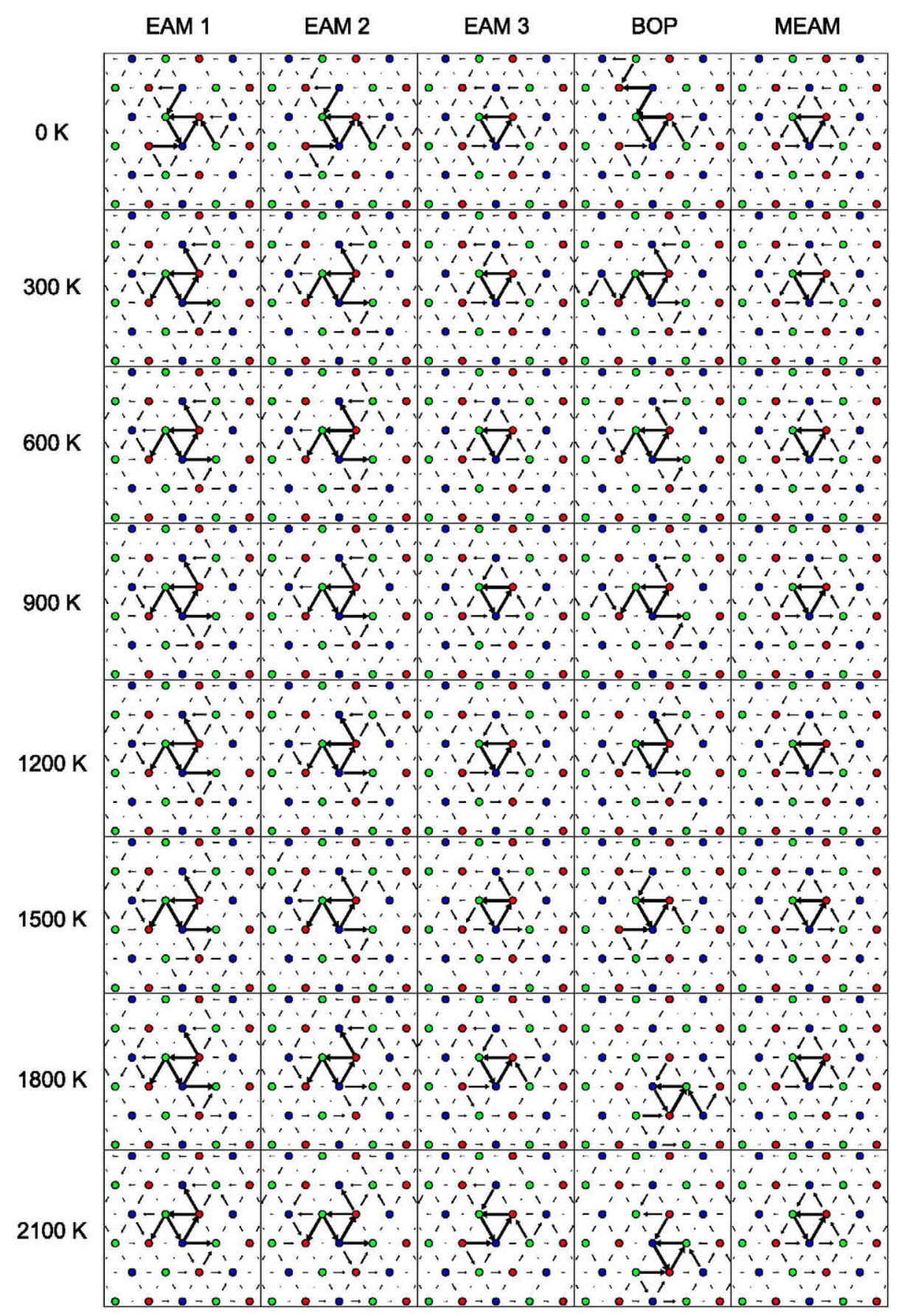

Figure 7. Time-averaged core structures for the five potentials tested here in the entire temperature range.

particular, they derived the so-called 'first-nearest-neighbor (1NN) inter-string interaction law' of the potential and used this in a 2D Frenkel-Kontorova (2D-FK) model of interacting $\langle 111\rangle$ strings to find the minimum energy screw core structure. Here, we extend their methodology to finite temperatures using the quasiharmonic approximation, i.e. by relating volume changes to temperature via pre-computed thermal expansion coefficients for each potential. In this fashion, we first compute the inter-string interaction laws as a function of the lattice parameter and then obtain the equivalent temperature as $T=3\left(a / a_{0}-1\right) / \alpha$. Here, $\alpha$ is the thermal expansion coefficient (given for each potential in table 1), $a_{0}$ is the lattice parameter at $0 \mathrm{~K}$ and $a$ is the lattice parameter corresponding to a temperature $T$ (within the quasiharmonic approximation). For the reminder of this section, we refer to $a$ as $a(T)$ to highlight this temperature dependence.

For each temperature $T$ the $1 \mathrm{NN}$ inter-string interaction law $U_{1}(d)$ was derived by rigidly translating a single $\langle 111\rangle$ string with respect to a perfect lattice with lattice parameter $a(T)$ and measuring the associated variation in energy under the particular interatomic potential. The resulting curve, which, according to the 2D-FK model defined in [30], is dominated by the contributions from the moving string interacting with its six 1NNs, can be unfolded using a Fourier 

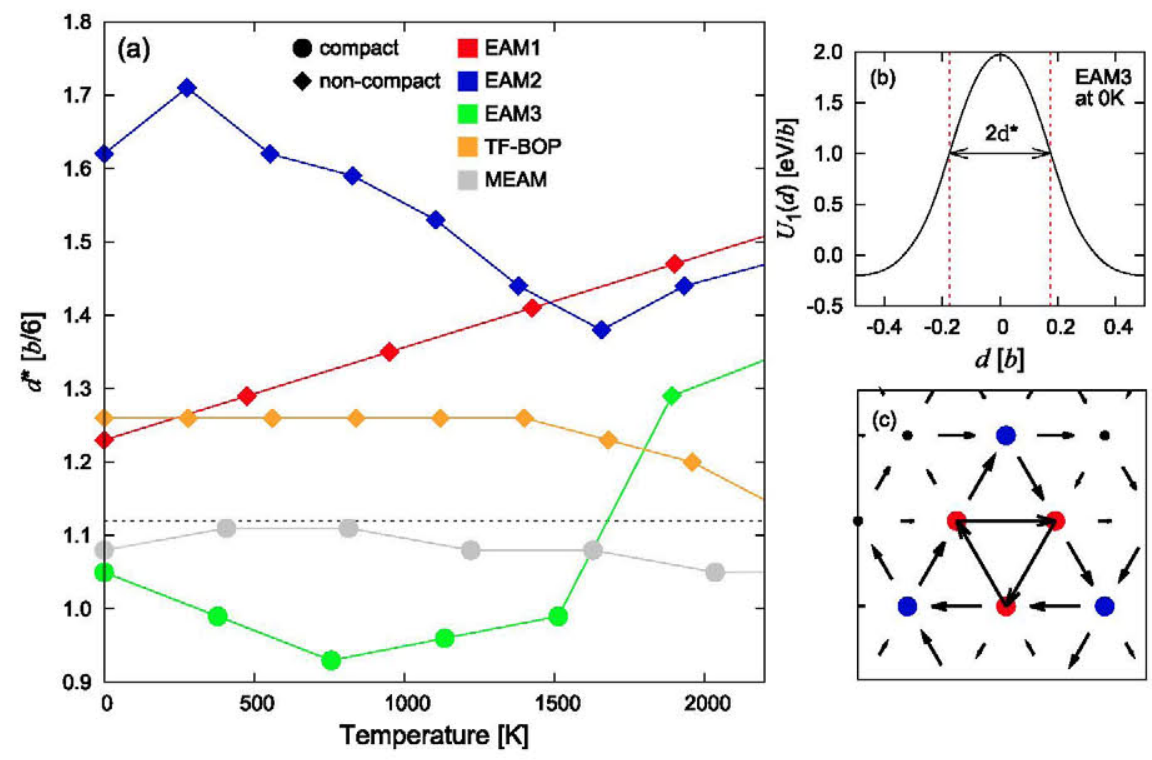

Figure 8. (a) Analysis of the favored core structure for the five different potentials as a function of temperature. A compact core is designated by a circle, and a non-compact one by a diamond. The $y$-position of each point is the magnitude of the distance between inflection points in the $1 \mathrm{NN}$ string-interaction law (i.e. $d^{*}$, which is the distance at which $\left.U_{1}^{\prime \prime}(d)=0\right)$ normalized to the quantity $b(T) / 6$, where $b(T)=\sqrt{3} a(T) / 2$. The dashed horizontal line separates the region of phase space where compact cores are favored (below) from the region where non-compact ones are favored (above). (b) The 1NN string-interaction law for EAM3 at $0 \mathrm{~K}$. The red dashed vertical lines indicate the positions of the inflection points in this curve. (c) Differential displacement map of the compact core predicted at $0 \mathrm{~K}$ for the $U_{1}$ function from EAM3. Each of the three strings closest to the core (red circles) is separated from its two closest secondary strings (blue circles) by $b / 6$. In the figure the temperature dependence of $b$ is omitted for the sake of clarity.

analysis to produce the required pair-wise interaction law for the 2D-FK model. An example of such a law for EAM3 at $0 \mathrm{~K}$ is shown in figure 8(b). A perfect screw dislocation, inserted into a lattice of $\langle 111\rangle$ atomic strings (for a given $a \equiv a(T)$ ) according to the isotropic elasticity solution, was then relaxed using $U_{1}(d)$ and the nature of the relaxed core was determined by visual inspection of its differential displacement map.

Figure 8(a) shows the variation in the favored core structure as a function of $T$ for each of the five potentials. On the $y$-axis of the plot we have calculated the ratio of the string separation $d^{*}$ associated with the inflection points in the corresponding $U_{1}(d)$ law (highlighted by the vertical dotted lines in figure $8(\mathrm{~b})$ ) to $b(T) / 6$, where $b(T)$ is the corresponding Burgers vector of each potential as a function of $T$.

As observed by Gilbert and Dudarev [30], the favored core structure depends on the positions of the inflection points of the $U_{1}(d)$ function. Specifically, a fully compact core is characterized by minimum string separations of $b(T) / 6$, which are the in-line separation distances between each of the three $\langle 111\rangle$ strings immediately surrounding the core (red circles in figure $8(\mathrm{c})$, which shows a differential displacement map for a compact core) and their two nearest strings forming the next shell of strings out from the core (blue circles in figure $8(\mathrm{c})$ ). When the inflection points in $U_{1}(d)$ are located at a distance of less than or equal to $b(T) / 6$, then the compact, non-dissociated core is always stable. Furthermore, even if the separations $d^{*}$ associated with $U_{1}^{\prime \prime}(d)=0$ are such that $\left|d^{*}\right|$ is somewhat greater than $b(T) / 6$, the compact core may still be stable provided that $U_{1}$ is only slowly varying around these inflection points-meaning that the forces $\left(-U_{1}^{\prime}(d)\right)$ between strings are relatively constant over a range of $d$ values. This, for example, is the situation in the case of the MEAM potential in figure $8(\mathrm{a})$, where the ratio $d^{*} /(b(T) / 6)$ is greater than one for all $T$, but the favored core remains compact.

However, when the ratio is significantly greater than one, as is the case for both EAM1 and EAM2 at all temperatures, then the compact core becomes unstable and the secondary strings out from the core tend to move towards (along $\langle 111\rangle$ ) one of their primary-string neighbors (signified by the major arrows in the 'arms' of the non-compact core shown for EAM1 in table 1), ultimately leading to the stabilization of the non-compact, three-fold symmetric dissociated core.

Thus, if, as a function of $T$, there is significant variation in this ratio, then the preferred equilibrium core structure can also change. In our analysis, we find that, consistent with the transition observed in figure 7 , there is a large shift in the value of $d^{*} /(b(T) / 6)$ for EAM3 $\left(d^{*}\right.$ is such that $U_{1}^{\prime \prime}\left(d^{*}\right)=0$ ) above $\sim 1500 \mathrm{~K}$. At this point, the equilibrium core structure diverges from the compact core, and becomes more and more dissociated as the temperature increases further. We discuss the implications of our findings in section 4 .

\section{Discussion}

\subsection{Comparison of the static properties of the potentials}

The five interatomic potentials tested here follow different formulations and have been fitted to different physical properties. It is not our objective to discuss the fitting process or the quality of each one, but only to discuss their 
performance in relation to screw dislocation modeling. When selecting potentials for screw dislocation simulations, two of the properties most looked at are the core structure and the Peierls stress. For W, these have been obtained using electronic structure calculations of different sorts, which reveal a compact core and a $\sigma_{\mathrm{P}}$ between 1.7 and $2.8 \mathrm{GPa}$. Regarding the core structure at $0 \mathrm{~K}$, only EAM3 and MEAM reproduce it correctly, although, as shown in section 3.2, the EAM3 potential does not preserve this structure at high temperature. In terms of $\sigma_{\mathrm{P}}$, the five potentials studied here give a range of values from 1.1 to $4.0 \mathrm{GPa}$. As table 1 shows, EAM3 and MEAM display values of 1.8 and $3.2 \mathrm{GPa}$, respectively, which are the most consistent with the range obtained by DFT calculations.

Furthermore, the subspaces of the energy landscape most relevant to screw dislocation motion are the Peierls potential and the $\gamma$ surface (figures 1 and 2). Relative to the DFT calculations, the sinusoidal shape of the Peierls potential is best reproduced by the EAM3 and MEAM potentials. Interestingly, however, the magnitude of the Peierls energy is most closely predicted by EAM2. EAM1 overestimates this energy by about a factor of two, while the TF-BOP predicts a trajectory with metastable states along its path, both in clear in disagreement with DFT calculations. Regarding the $\gamma$ surface, the EAM1, EAM3 and MEAM potentials all predict the essential qualitative and quantitative features of the DFT results and are also in good agreement with the results by Gröger et al using a TB-BOP [29].

Thus, on the basis of all these calculations, the MEAM potential appears to be the best suited of those tried here to carry out dislocation simulations at any temperature. When computational cost is of the essence, EAM3 may be considered an acceptable replacement for static calculations or for calculations at low temperatures and stresses.

\subsection{Mobility of screw dislocations}

Dislocation mobility is highly multidimensional in that it displays multiparametric dependences, e.g. on stress, temperature, dislocation character, slip system, etc. Dislocation velocities are difficult to infer from straining experiments, while they are costly and subject to size limitations in simulations. Measurements [31] and calculations [32, 33] of edge dislocation velocities have been carried out in W. However, other than the values computed by Tian and Woo [9] at very high stress (>3.6 GPa), to the best of our knowledge no data exist on screw dislocation mobility in $W$ at low stresses. In this work, we have focused on the temperature and stress dependences, while we have kept the dislocation character and the slip system fixed.

A quick look at figure 5 reveals several interesting details. First, the EAM2 and MEAM consistently give the highest and lowest velocities, respectively, regardless of temperature. Since atomistic simulations commonly overestimate screw dislocation velocities, particularly in the low-stress range, this may be another reason in favor of using MEAM. This is likely to be due to the fact that the dislocation core remains compact in the entire temperature range using the
MEAM potential (cf figure 7). Second, screw dislocations move by thermally-activated mechanisms below the Peierls stress, transitioning to a viscous damping regime above it. At the maximum applied stress of $2000 \mathrm{MPa}$, some dislocations have been driven past the Peierls stress as given by their respective potentials (cf table 1 ). This is certainly the case for the TF-BOP and possibly the EAM2 and EAM3 potentials. One would therefore expect to see a gradual exhaustion of the thermally-activated regime and a transition into a linear regime. Interestingly, such a transition appears to occur for the EAM1 potential which has $\sigma_{\mathrm{P}}=4.0 \mathrm{GPa}$. It was shown by Gilbert et al [13], however, that the actual transition stress decreases with the square of the temperature, which may be what is seen here. Appropriate exponential fits to the data shown in figure 5 carried out in the appendix reveal useful parameters that define the thermally-activated regime.

\subsection{Dislocation core transformation}

The behavior that emanates from the results in figures 5 and 6 for the EAM3 potential is the manifestation of a temperature-driven dislocation core transformation that occurs as a result of changes to the free energy landscape. We have characterized this transformation via differential displacement maps of time-averaged atomic positions at finite temperatures, and a quasiharmonic analysis of the locations of the inflection points in the $\langle\mathbf{1 1 1}\rangle$ interaction energy, which is known to control the dislocation core structure (cf figures 6 and 8).

This is seen to affect the dislocation mobility as well. We have shown that the reported core transformation has an impact on both the stress and the temperature dependence. Indeed, in the analysis carried out in the appendix, it is shown that the temperature dependence of the fitted $\sigma-v$ relations at $T<1200 \mathrm{~K}$ for EAM3 does not carry over to higher temperatures. By contrast, the same analysis does not yield significant differences between the low and high temperature regimes for the EAM1 and MEAM potentials. This is further indication that the core structure may impact the motion mechanisms in the corresponding temperature range.

We emphasize, however, that as long as there does not exist independent evidence of this dislocation core structural change with temperature, the discussion about its true impact on the dynamic behavior of screw dislocations remains solely speculative, and we cautiously warn against using the EAM3 potential above the observed transformation temperature of $\approx 1500 \mathrm{~K}$. In this sense, the quasiharmonic analysis performed in section 3.3 would be very amenable to DFT calculations, as it consists solely of zero-temperature calculations. This would provide an independent means to prove or disprove - at least within the limitations of the quasiharmonic analysis-the behavior predicted by EAM3 at high temperature.

\subsection{Mechanism of motion}

The dislocation trajectories shown in figure 4 demonstrate that screw dislocations move primarily along the direction 
of the applied stress. The only notable exception is the TF-BOP, for which significant transitions out of plane are observed. The figure, however, does not provide insights into the atomistic mechanism of motion. Then, in figure 6 trajectories for the EAM3 were analyzed with higher spatial and temporal resolution at temperatures of 600 and $1800 \mathrm{~K}$. At both temperatures, the dislocation moves by elementary $\{110\}$ kink-pair episodes. It is reasonable to assume that this mechanism can be extrapolated to other potentials that yield similar effective trajectories (close to the MRSS plane). However, for the EAM3 results, there are some differences in terms of the temperature at which the trajectory was extracted. At $1800 \mathrm{~K}$ it appears as though the unit mechanism is composed of one $+30^{\circ}$ jump (on a $(01 \overline{1}$ ) plane) followed by a correlated $-30^{\circ}$ jump (on a (10 $\overline{1}$ ) plane). In other words, the dislocation appears to move by kink-pair episodes on the $(11 \overline{2})$ plane that consist of two alternating and correlated $\pm 30^{\circ}$ kinks. This is consistent with the mechanism proposed by Duesbury [34]. Overall, this results in a trajectory that follows a random walk and that, on average, forms zero degrees with the MRSS plane. Interestingly, at $600 \mathrm{~K}$ kink pairs on the $+30^{\circ}$ plane seem to be favored in a proportion of three or more to one over $-30^{\circ}$ ones. It is unclear at this point whether the dislocation core transition discussed above for EAM3 is responsible for this difference. Again, as stated in section 4.3, we are reluctant to construe this as real physical behavior until more is known about the core structure transformation. Our main message from the analysis of trajectories is that despite the MRSS plane being of the $\{112\}$ family, motion proceeds by way of kink pairs on $\{110\}$ planes, presumably for all potentials.

\section{Summary}

To summarize, the main findings of this paper can be condensed into the following main items.

- We have calculated static properties relevant to screw dislocations using five different interatomic potentials for W. These include three EAM, one BOP and one MEAM.

- We have calculated screw dislocation mobilities for all potentials on a $\{112\}$ glide plane. Our calculations provide elements to judge the MEAM potential as the most suitable one for dislocation calculations.

- We have observed a temperature-induced dislocation core transformation-from compact to dissociated-for one of the potentials tested. Lacking independent confirmation, we cannot confirm whether this corresponds to a real physical phenomenon or is an artifact, but the transformation is indeed seen to impact the dynamic properties of dislocations.

- Our analysis of the five interatomic potentials suggests, first, that the atomistic nature of the dislocation core governs the behavior at larger scales and, second, a purely static treatment of the dislocation core is insufficient to predict and describe the dynamics of dislocations.

\section{Acknowledgments}

We thank D Terentyev for assisting in the calculation of the Peierls stress for EAM2. This work was performed under the auspices of the US Department of Energy by Lawrence Livermore National Laboratory under Contract DE-AC5207NA27344. We specifically acknowledge support from the Laboratory Directed Research and Development Program under project 11-ERD-023. DC and JMP acknowledge support from the 7th Framework Programme with project HiPER: European High Power Laser Energy Research Facility, Grant Agreement No. 211737. We specifically acknowledge the $\mathrm{PhD}$ program support from Universidad Politécnica de Madrid. This work was also partly funded by the RCUK Energy Programme under grant EP/I501045 and the European Communities under the contract of Association between EURATOM and CCFE. The views and opinions expressed herein do not necessarily reflect those of the European Commission.

\section{Appendix}

Here we analyze the overall impact of the dislocation core transition for the EAM3 potential on dislocation mobility. We fit the data given in figure 3 to the general expression

$$
v(\sigma, T)=A \sigma \exp \left(-\frac{H_{0}-\sigma V^{*}}{k T}\right),
$$

where $A, H_{0}$ and $V^{*}$ are fitting constants that represent, respectively, a velocity prefactor, the kink-pair energy at $0 \mathrm{~K}$ and the activation volume. We obtain these for three potentials, namely, EAM1, EAM3 and MEAM, and carry out the fit first including all temperatures. The results for each case are shown in table A.1. These values deserve some commentary, particularly $H_{0}$ and $V^{*}$. Using the method described by Ventelon and Willaime [25], we have obtained a kink-pair energy of $H_{0}=1.7 \mathrm{eV}$ for the MEAM potential. Experimentally, Brunner [39] has obtained a value of $1.75 \mathrm{eV}$ from the temperature dependence of flow stress measurements in $\mathrm{W}$, in very good agreement with the calculated value but significantly higher than the MD values. Giannattasio et al [40] have obtained values of the order of $1.0 \mathrm{eV}$ inferred from the strain rate dependence of the brittle-to-ductile transition, still much larger than those reported here. Similarly, Tarleton and Roberts [41] have found values of $V^{*}=20 \mathrm{~b}^{3}$ to be representative of the kink-pair process in W. Again, these are two orders of magnitude larger than ours. The magnitudes of $H_{0}$ and $V^{*}$ obtained in our analysis suggest a very 'soft' thermally-activated process, something not necessarily consistent with the static data presented in section 2.1. The low values of $H_{0}$ and $V^{*}$ obtained in our simulations are likely due to overdriven screw dislocation dynamics in the MD simulations.

Next, we obtain additional fits using only data at 300 , 600 and $900 \mathrm{~K}$, i.e. at temperatures below the presumed core transformation for the EAM3 potential. The corresponding parameter values are shown in bold script for each case in table A.1. The percentage difference between the values 
Table A.1. Fitting parameters for the analytical mobility function (A.1). The average fitting errors for $A, H_{0}$ and $V^{*}$ were, respectively, $6 \%$, $9 \%$ and $10 \%$. Regular script: values from full temperature fits; bold script: values from low temperature $(\leq 900 \mathrm{~K})$ fits; in parentheses: percentage difference between the two sets of fits.

\begin{tabular}{llll}
\hline Potential & $A\left(\mathrm{~ms}^{-1} \mathrm{MPa}^{-1}\right)$ & $H_{0}(\mathrm{eV})$ & $V^{*}\left(\mathrm{~b}^{3}\right)$ \\
\hline EAM3 & $0.26 \mathbf{0 . 2 4}(8 \%)$ & $0.05 \mathbf{0 . 0 4}(20 \%)$ & $0.42 \mathbf{0 . 1 9}(55 \%)$ \\
MEAM & $0.19 \mathbf{0 . 1 7}(10 \%)$ & $0.08 \mathbf{0 . 0 7}(12 \%)$ & $0.26 \mathbf{0 . 2 3}(12 \%)$ \\
EAM1 & $0.30 \mathbf{0 . 2 8}(7 \%)$ & $0.08 \mathbf{0 . 0 7}(12 \%)$ & $0.24 \mathbf{0 . 2 4}(0 \%)$ \\
\hline
\end{tabular}

for full and low temperature fits is given in parentheses. Although the differences in the parameter $A$ are similar in all cases, those for $H_{0}$ and $V^{*}$ are clearly largest for EAM3. in particular, the differences in the activation volume, which is known to be most sensitive to the core structure, are considerable. This further reinforces the notion that, from a dynamical standpoint, dislocations are behaving differently below and above $\approx 1000 \mathrm{~K}$. In contrast, for the other two potentials, the dynamic behavior above and below this presumed transition temperature is governed by the same laws. The low temperature fits obtained here are shown in figure 5 . The fits provide very good agreement with the EAM1 and MEAM data at all temperatures, whereas they gradually worsen for EAM3 as the temperature increases. Moreover, the deviation of the fits above $\sigma_{\mathrm{P}}$ for the EAM3 potential can be clearly appreciated.

\section{References}

[1] Zinkle S J and Ghoniem N M 2011 J. Nucl. Mater. 4172

[2] Lassner E and Schubert W F 1999 Tungsten: Properties, Chemistry, Technology of the Element, Alloys, and Chemical Compounds (New York: Kluwer)

[3] Romaner L, Ambrosch-Draxl C and Pippan R 2010 Phys. Rev. Lett. 104195503

[4] Wurster S, Gludovatz B and Pippan R 2010 Int. J. Refract. Hard Mater. 28692

[5] Li H, Wurster S, Motz C, Romaner L, Ambrosch-Draxl C and Pippan R 2012 Acta Mater: 60748

[6] Samolyuk G D, Osetsky Y N and Stoller R E 2013 J. Phys.: Condens. Matter 25025403

[7] Mrovec M, Gröger R, Bailey A G, Nguyen-Manh D, Elsässer C and Vitek V 2007 Phys. Rev. B 75104119

[8] Fikar J, Schäublin R and Björkas C 2009 Adv. Mater. Res. 59247

[9] Tian X and Woo C 2004 Mater. Sci. Eng. A 369210

[10] Mrovec M 2011 private communication

[11] Argon A S and Maloof S R 1966 Acta Metall. 141449

[12] Chaussidon J, Fivel M and Rodney D 2006 Acta Mater $\mathbf{5 4} 3407$
[13] Gilbert M R, Queyreau S and Marian J 2011 Phys. Rev. B 84174103

[14] Cereceda D, Perlado J M and Marian J 2012 Comput. Mater. Sci. 62272

[15] Plimpton S J 1995 J. Comput. Phys. 1171

[16] Knibbs R H 1969 J. Sci. Instrum. 2515

[17] Zhou X W et al 2001 Acta Mater. 494005

[18] Ackland G J and Thetford R 1987 Phil. Mag. A 5615

[19] Juslin N, Erhart P, Träskelin P, Nord J, Henriksson K O E, Nordlund K, Salonen E and Albe K 2005 J. Appl. Phys. 98123520

[20] Park H, Fellinger M R, Lenosky T J, Tripton W W, Trinkle D R, Rudin S P, Woodward C, Wilkins J W and Hennig R G 2012 Phys. Rev. B 85214121

[21] Gordon P A, Neeraj T, Li Y and Li J 2010 Modelling Simul. Mater. Sci. Eng. 18085008

[22] Duesbery M S and Vitek V 1998 Acta Mater. 461481

[23] Henkelman G, Johannesson G and Jonsson H 2000 Progress on Theoretical Chemistry and Physics (Dordrecht: Kluwer) pp 269-300

[24] Gröger R and Vitek V 2012 Modelling Simul. Mater. Sci. Eng. 20035019

[25] Ventelon L and Willaime F 2007 J. Comput. Aided Mater. Des. 1485

[26] Ventelon L and Willaime F 2013 in preparation

[27] Bulatov V V and Cai W 2008 Computer Simulation of Dislocations (Oxford: Oxford University Press)

[28] Olmsted D L, Hector L G, Curtin W A and Clifton R J 2005 Modelling Simul. Mater. Sci. Eng. 13371

[29] Gröger R, Racherla V, Bassani J L and Vitek V 2008 Acta Mater. 565412

[30] Gilbert M R and Dudarev S L 2010 Phil. Mag. 901035

[31] Schadler H W 1964 Acta Metall. 12861

[32] Gumbsch P and Gao H 1999 Science 283965

[33] Li Q and Shi S-Q 2002 Appl. Phys. Lett. 803069

[34] Duesbery M S, unpublished

[35] Malerba L et al 2010 J. Nucl. Mater. 40619

[36] Ercolessi F and Adams J B 1994 Europhys. Lett. 26583

[37] Rodney D and Proville L 2009 Phys. Rev. B 79094108

[38] Finnis M W and Sinclair J E 1984 Phil. Mag. A 5045

[39] Brunner D 2000 Mater: Trans. JIM 41152

[40] Giannattasio A, Tanaka M, Joseph T D and Roberts S G 2007 Phys. Scr. T128 87

[41] Tarleton E and Roberts S G 2009 Phil. Mag. 892759 